\title{
B-hadron production in top quark decay
}

\author{
Gennaro Corcella* \\ Dipartimento di Fisica \\ Università di Roma 'La Sapienza' \\ P. le A.Moro 2, I-00185 Roma, Italy \\ E-mail: Gennaro.Corcella@romal.infn.it

\section{Volker Drollinger} \\ Dipartimento di Fisica Galileo Galilei \\ Università di Padova and INFN, Sezione di Padova \\ Via Marzolo 8, I-35131 Padova, Italy \\ E-mail: Volker.Drollinger@cern.ch
}

We present the energy distribution of $b$-flavoured hadrons in top quark decay using the PYTHIA and HERWIG event generators, which we tune to LEP and SLD data. We find that fitting the string and cluster models is essential to reproduce the $e^{+} e^{-}$data and to reliably predict $B$-hadron production in top decay. We also compare the PYTHIA and HERWIG results with the ones yielded by resummed calculations based on the fragmentation function formalism.

International Workshop on Top Quark Physics

January 12-15, 2006

Coimbra, Portugal

\footnotetext{
${ }^{*}$ Speaker.
} 
Top quark phenomenology is one of the main fi elds of investigation in theoretical and experimental particle physics. The experiments at the Tevatron accelerator and, in future, at the LHC will allow one to perform improved measurements of the top properties, such as its mass, thanks to the production of large amounts of $t \bar{t}$ pairs.

In this paper we study bottom quark fragmentation in top quark decay $(t \rightarrow b W)$, which is responsible of one of the largest contribution to the uncertainty on the top mass measurement at the Tevatron [1, 2] and the LHC [3]. In particular, in the analysis of Ref. [4] the top quark mass is determined using at the LHC fi nal states with leptons and $J / \psi$ 's, where the leptons come from the $W$ decay $W \rightarrow \ell v$, and the $J / \psi$ 's from the decay of a $b$-flavoured hadron $B$. In [4] the PYTHIA event generator [5] was exploited, and the error on $m_{t}$ was estimated to be $\Delta m_{t} \simeq 1 \mathrm{GeV}$, with $b$-fragmentation being the largest source of uncertainty. In [6], the invariant mass $m_{B \ell}$, yielded by the HERWIG [7] event generator, was used to fit $m_{t}$, and the impact of matrix-element corrections to the simulation of top decay [8] was investigated.

Bottom quark fragmentation in top decay was studied in $[9,10]$, following the method of perturbative fragmentation functions [11]. The NLO $b$-quark energy spectrum is expressed as the convolution of a coeffi cient function, describing the emission of a massless parton, and a perturbative fragmentation function $D\left(m_{b}, \mu_{F}\right)$, associated with the transition of a massless parton into a massive $b . D\left(m_{b}, \mu_{F}\right)$ follows the Dokshitzer-Gribov-Lipatov-Altarelli-Parisi (DGLAP) evolution equations $[13,14]$, which can be solved once an initial condition at a scale $\mu_{0 F}$ is given. The initial condition of the perturbative fragmentation function, fi rst computed in [11], was proved to be process-independent in [12]. Solving the DGLAP evolution equations we can resum the large $\ln \left(m_{t}^{2} / m_{b}^{2}\right)$ which appears in the NLO massive $b$-spectrum (collinear resummation). Both the top-decay coeffi cient function, computed in [9], and the initial condition $D\left(m_{b}, \mu_{0 F}\right)$ present terms which become large when the $b$-quark energy fraction $x_{b}$ approaches 1 , which corresponds to soft-gluon radiation. Soft contributions in the initial condition (process independent) and in the coeffi cient function (process dependent) were resummed in the NLL approximation in [12] and [10], respectively. In order to predict the spectrum of $b$-flavoured hadrons, perturbative calculations need to be supplement by non-perturbative models. In $[9,10]$, the $B$-hadron spectrum in top decay was presented, after fi tting a few hadronization models to SLD [15] and ALEPH [16] data.

Following the lines of [17], in this paper we would like to address $b$-fragmentation in top decay, using the PYTHIA and HERWIG event generators. As discussed in [17], PYTHIA and HERWIG simulate multiple radiation in top decay in the soft or collinear approximation, and are provided with matrix-element corrections $[8,18]$ to allow hard and large-angle emission. The hadronization mechanism is simulated by the string model [19] in PYTHIA, and by the cluster model [20] in HERWIG.

For the sake of a reliable prediction of the $B$-energy distribution in $t \rightarrow b W$, we need to use models and parametrizations which are able to describe well the $B$-spectrum at $e^{+} e^{-}$machines. We consider ALEPH [16], OPAL [21] and SLD [15] data on the $B$ energy fraction $x_{B}$ in $Z \rightarrow b \bar{b}$ events, where $x_{B}$ is defi ned as follows:

$$
x_{B}=\frac{2 p_{B} \cdot p_{Z}}{m_{Z}^{2}},
$$

with $p_{Z}$ and $p_{B}$ being the $Z$ and $B$ momenta, respectively. 
Table 1: Parameters of HERWIG and PYTHIA hadronization models tuned to $e^{+} e^{-}$data, along with the $\chi^{2}$ per degree of freedom.

\begin{tabular}{|c|c|}
\hline HERWIG & PYTHIA \\
\hline \hline CLSMR $(1)=0.4$ & \\
\hline CLSMR $(2)=0.3$ & PARJ(41) $=0.85$ \\
\hline DECWT $=0.7$ & PARJ $(42)=1.03$ \\
\hline CLPOW $=2.1$ & PARJ $(46)=0.85$ \\
\hline PSPLT $(2)=0.33$ & \\
\hline \hline$\chi^{2} /$ dof $=222.4 / 61$ & $\chi^{2} /$ dof $=45.7 / 61$ \\
\hline
\end{tabular}

We use the versions HERWIG 6.506 and PYTHIA 6.220, and fi nd that the default parametrizations are unable to fi t such data, as one gets $\chi^{2} / \mathrm{dof}=739.4 / 61$ (HERWIG) and $\chi^{2} / \mathrm{dof}=467.9 / 61$ (PYTHIA). As in [17], we tune the cluster and string models to the data, while we leave unchanged the parameters of HERWIG and PYTHIA which are related to the perturbative phase of the parton showers. Our best fi ts are summarized in Table 1: for PYTHIA we are able to fi nd a parametrization which is able to reproduce well the data $\left(\chi^{2} /\right.$ dof $\left.=45.7 / 61\right)$; HERWIG, even after the fi t, is still not able to describe the $x_{B}$-spectrum very well, but the comparison is anyway much better than with the default parameters $\left(\chi^{2} / \mathrm{dof}=222.4 / 61\right)$. We have also checked that the parametrizations in Table 1 work well for the new model implemented in PYTHIA 6.3 [22]. The HERWIG and PYTHIA spectra, before and after the fit, along with the experimental data, are presented in Figures 1 and 2. For the sake of comparison, we also show the $x_{B}$-spectrum yielded by the NLO+NLL calculation of Ref. [12], convoluted with the Kartvelishvili hadronization model [23]:

$$
D^{\mathrm{np}}(x ; \gamma)=(1+\gamma)(2+\gamma)(1-x) x^{\gamma}
$$

We fit the model (2) to the data in the range $0.18 \leq x_{B} \leq 0.94$, to avoid the regions at small and large $x_{B}$, where, as pointed out in [12], the resummed calculation yields a negative distribution. Setting $m_{Z}=91.118 \mathrm{GeV}, m_{b}=5 \mathrm{GeV}$ and $\Lambda \frac{(5)}{\mathrm{MS}}=200 \mathrm{GeV}$ in the perturbative calculation, we get $\gamma=17.178 \pm 0.303$ From Figs. 1 and 2 we learn that default HERWIG and PYTHIA are far from the data. After the tuning, PYTHIA reproduces the data quite well, while HERWIG yields a broader distribution; the resummed calculation is consistent with the data. and $\chi^{2} / \mathrm{dof}=46.2 / 53$ from the fit.

Using the parametrizations in Table 1 , we can predict the $B$-energy distribution in $t \rightarrow b W$, which will be expressed in terms of the variable

$$
x_{B}=\frac{1}{1-w} \frac{2 p_{B} \cdot p_{t}}{m_{t}^{2}}
$$

where $p_{t}$ is the top momentum and $1 /(1-w)$ is a normalization factor, with $w=1-m_{W}^{2} / m_{t}^{2}+$ $m_{b}^{2} / m_{t}^{2}$. In Fig. 3 we present the $B$-spectrum in top decay according to HERWIG, PYTHIA and the resummed calculation of $[9,10]$, convoluted with the Kartvelishvili model. The comparison exhibited in Fig. 3 shows similar features to Figures 1 and 2, and reflects the quality of the fi ts to 


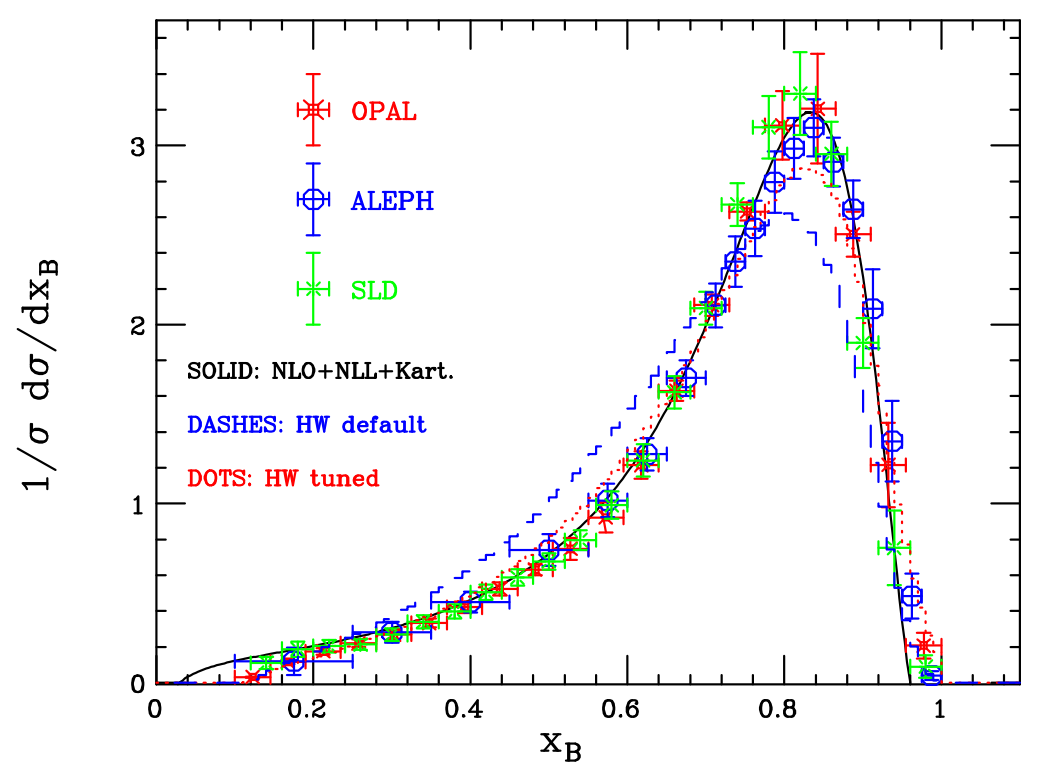

Figure 1: Data from LEP and SLD experiments, compared with the NLO+NLL calculation convoluted with the Kartvelishvili model (solid) and HERWIG 6.506, using the default parametrization (dashed) and our tuning (dotted).

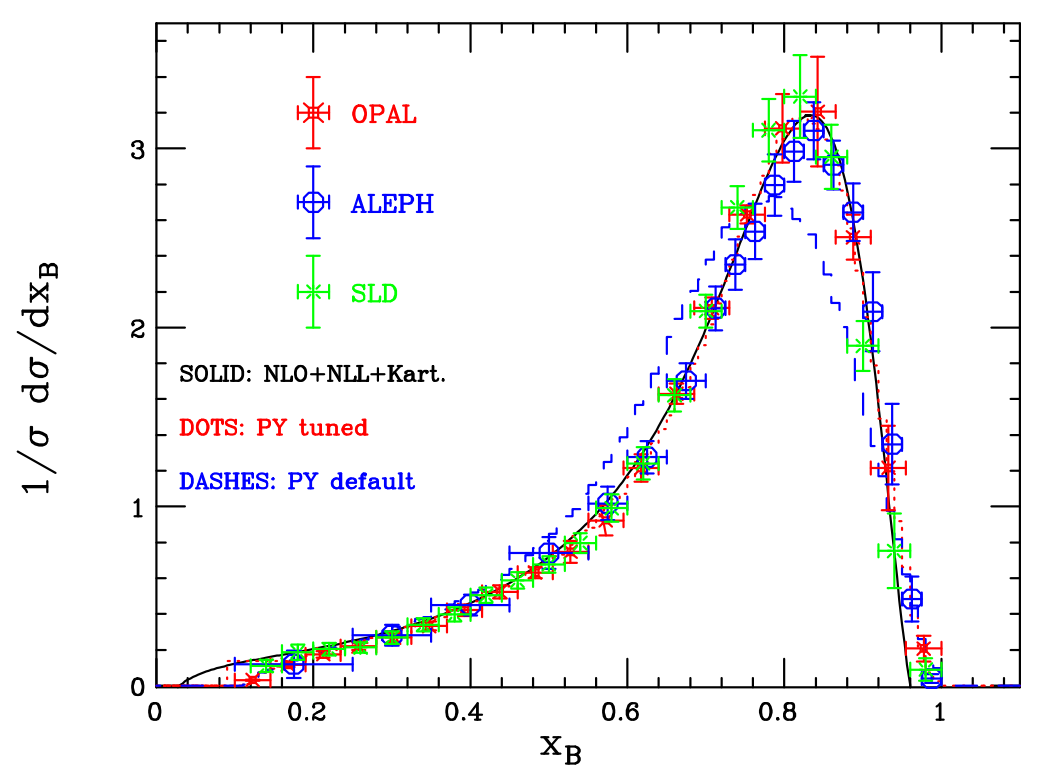

Figure 2: As in Fig. 1, but comparing data and the NLO+NLL calculation with default (dashed) and tuned (dotted) PYTHIA 6.220.

the $e^{+} e^{-}$data: PYTHIA reproduces the peak of the resummed calculation rather well, while it is below the NLL prediction at $x_{B}<0.7$, and above at $x_{B}>0.9$. HERWIG is below the resummed spectrum in most the $x_{B}$-range, and above it only at large $x_{B}$.

Finally, we wish to present results in Mellin moment space, making use of the data reported by the DELPHI Collaboration in [24] on the moments of the $B$ cross section in $e^{+} e^{-}$processes. From the point of view of resummed calculations, working in moment space presents several advantages: 


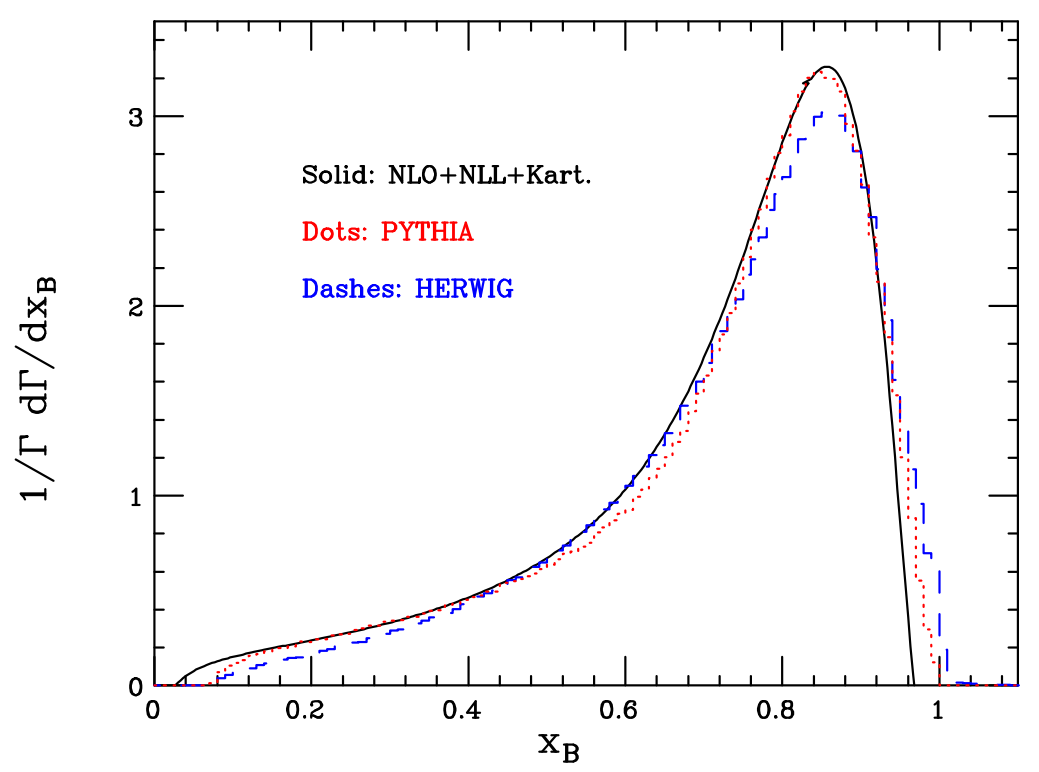

Figure 3: $B$-hadron spectra in top decay, for $m_{t}=175 \mathrm{GeV}$, according to a NLO+NLL computation convoluted with the Kartvelishvili model (solid line), HERWIG (dashed) and PYTHIA (dotted).

in $N$-space, convolutions become ordinary products and the relation between parton- and hadronlevel moments is just $\sigma_{N}^{B}=\sigma_{N}^{b} D_{N}^{\mathrm{np}}$, where $D_{N}^{\mathrm{np}}$ is the non-perturbative fragmentation function in Mellin space. Therefore, there is no need to introduce a functional form for the hadronization model in $x_{B}$-space. Also, $N$ - spectra are well defi ned, and do not present the problems of the $x_{B}$-results, which are negative at small or large $x_{B}$.

In Table 2 we compare the DELPHI moments with the ones given by the tuned versions of HERWIG and PYTHIA, and the predictions for top decay. We also quote the results yielded by the NLL calculations of Refs. [9, 10], extracting $D_{N}^{\text {np }}$ from the data. As for $e^{+} e^{-} \rightarrow b \bar{b}$ processes, the moments given by HERWIG and PYTHIA are consistent, within the error ranges, with the DELPHI ones. It is interesting that HERWIG is compatible with the DELPHI moments, even though it was only marginally consistent with LEP and SLD data in $x_{B}$-space. The results for top decay have similar features to the $x_{B}$-spectra: PYTHIA is very close to the NLL calculation, which uses $D_{N}^{\mathrm{np}}$ extracted from the DELPHI data, while HERWIG, whose predictions are shifted toward larger $x_{B}$, gives larger moments.

In summary, we reviewed recent results on $b$-flavoured hadron production in top quark decay. We tuned HERWIG and PYTHIA to LEP and SLD data and presented results on the $B$-hadron spectrum in top decay in both $x_{B}$ and moment spaces. In fact, fi tting the cluster and string model turned out to be necessary to reproduce the $e^{+} e^{-}$data. The results were also compared with resummed calculations, based on the fragmentation function formalism. We think that our analysis and fi ts can be useful to improve the present understanding of $b$-quark fragmentation in top quark decay. It will be very interesting to investigate how the tuned versions of HERWIG and PYTHIA fare with respect to other observables. For example, it may be worthwhile reconsidering the studies in Refs. [4, 6] with the parametrizations which we have proposed, and estimate the contribution of $b$-fragmentation to the uncertainty on the top quark mass reconstruction. 
Table 2: Moments $\sigma_{N}^{B}$ from DELPHI [24], and moments in $e^{+} e^{-}$annihilation and top ( $t$ ) decay, using NLL resummed calculations, HERWIG (HW) and PYTHIA (PY).

\begin{tabular}{|c|cccc|}
\hline & $\langle x\rangle$ & $\left\langle x^{2}\right\rangle$ & $\left\langle x^{3}\right\rangle$ & $\left\langle x^{4}\right\rangle$ \\
\hline \hline$e^{+} e^{-}$data $\sigma_{N}^{B}$ & $0.7153 \pm 0.0052$ & $0.5401 \pm 0.0064$ & $0.4236 \pm 0.0065$ & $0.3406 \pm 0.0064$ \\
\hline \hline$e^{+} e^{-}$NLL $\sigma_{N}^{b}$ & 0.7801 & 0.6436 & 0.5479 & 0.4755 \\
\hline$D_{N}^{\mathrm{np}}$ & 0.9169 & 0.8392 & 0.7731 & 0.7163 \\
\hline$e^{+} e^{-} \mathrm{HW} \sigma_{N}^{B}$ & 0.7113 & 0.5354 & 0.4181 & 0.3353 \\
\hline$e^{+} e^{-} \mathrm{PY} \sigma_{N}^{B}$ & 0.7162 & 0.5412 & 0.4237 & 0.3400 \\
\hline \hline$t$-dec. NLL $\Gamma_{N}^{b}$ & 0.7883 & 0.6615 & 0.5735 & 0.5071 \\
\hline$t$-dec. NLL $\Gamma_{N}^{B}=\Gamma_{N}^{b} D_{N}^{\mathrm{np}}$ & 0.7228 & 0.5551 & 0.4434 & 0.3632 \\
\hline$t$-dec. $\mathrm{HW} \Gamma_{N}^{B}$ & 0.7325 & 0.5703 & 0.4606 & 0.3814 \\
\hline$t$-dec. PY $\Gamma_{N}^{B}$ & 0.7225 & 0.5588 & 0.4486 & 0.3688 \\
\hline
\end{tabular}

\section{References}

[1] CDF Collaboration, A. Abulencia et al., Phys. Rev. Lett. 96 (2006) 022004.

[2] D0 Collaboration, V.M. Abazov et al., Phys. Lett. B 606 (2005) 25.

[3] M. Beneke, I. Efthymiopoulos, M.L. Mangano, J. Womersley et al., in Proceedings of 1999 CERN Workshop on Standard Model Physics (and more) at the LHC, CERN 2000-004, G. Altarelli and M.L. Mangano eds., p. 419, hep-ph/0003033.

[4] A. Kharchilava, Phys. Lett. B 476 (2000) 73.

[5] T. Sjöstrand, L. Lönnblad and S. Mrenna, hep-ph/0108264.

[6] G. Corcella, J. Phys. G26 (2000) 634;

G. Corcella, M.L. Mangano and M.H. Seymour, JHEP 0007 (2000) 004.

[7] G. Corcella, I.G. Knowles, G. Marchesini, S. Moretti, K. Odagiri, P. Richardson, M.H. Seymour, B.R. Webber, JHEP 0101 (2001) 010.

[8] G. Corcella and M.H. Seymour, Phys. Lett. B442 (1998) 417.

[9] G. Corcella and A.D. Mitov, Nucl. Phys. B623 (2002) 247.

[10] M. Cacciari, G. Corcella and A.D. Mitov, JHEP 0212 (2002) 015.

[11] B. Mele and P. Nason, Nucl. Phys. B 361 (1991) 626.

[12] M. Cacciari and S. Catani, Nucl. Phys. B617 (2001) 253.

[13] G. Altarelli and G. Parisi, Nucl. Phys. B126 (1977) 298.

[14] L.N. Lipatov, Sov. J. Nucl. Phys. 20 (1975) 95; V.N. Gribov and L.N. Lipatov, Sov. J. Nucl. Phys. 15 (1972) 438; Yu.L. Dokshitzer, Sov. Phys. 46 (1977) 641.

[15] SLD Collaboration, K. Abe et al., Phys. Rev. Lett. 84 (2000) 4300.

[16] ALEPH Collaboration, A. Heister et al., Phys. Lett. B512 (2001) 30.

[17] G. Corcella and V. Drollinger, Nucl. Phys. B730 (2005) 82.

[18] E. Norrbin and T. Sjostrand, Nucl. Phys. B603 (2001) 297.

[19] B. Andersson, G. Gustafson, G. Ingelman, T. Sjöstrand, Phys. Rept. 97 (1983) 31. 
[20] B.R. Webber, Nucl. Phys. B238 (1984) 492.

[21] OPAL Collaboration, G. Abbiendi et al., Eur. Phys. J. C29 (2003) 463.

[22] T. Sjöstrand, L. Lönnblad, S. Mrenna and P.Z. Skands, hep-ph/0308153.

[23] V.G. Kartvelishvili, A.K. Likehoded and V.A. Petrov, Phys. Lett. B78 (1978) 615.

[24] DELPHI Collaboration, G. Barker et al., DELPHI 2002-069, CONF 603. 